\title{
BMJ Open Predictors and triggers of incivility within healthcare teams: a systematic review of the literature
}

\author{
Sandra Keller (D) , ${ }^{1}$ Steven Yule, ${ }^{1,2,3,4}$ Vivian Zagarese, ${ }^{5}$ Sarah Henrickson Parker ${ }^{5,6,7}$
}

To cite: Keller S, Yule S, Zagarese V, et al. Predictors and triggers of incivility within healthcare teams: a systematic review of the literature. BMJ Open 2020;10:e035471. doi:10.1136/ bmjopen-2019-035471

- Prepublication history and additional material for this paper are available online. To view these files, please visit the journal online (http://dx.doi org/10.1136/bmjopen-2019035471).

Received 02 November 2019

Revised 05 March 2020

Accepted 29 April 2020

Check for updates

(C) Author(s) (or their employer(s)) 2020. Re-use permitted under CC BY. Published by BMJ.

${ }^{1}$ Center for Surgery and Public Health (CSPH), Brigham and Women's Hospital, Boston, Massachusetts, USA ${ }^{2}$ STRATUS Center for Medical Simulation, Boston,

Massachusetts, USA

${ }^{3}$ Department of surgery, Harvard Medical School, Boston, Massachusetts, USA

${ }^{4}$ Department of Clinical Surgery, University of Edinburgh,

Edinburgh, United Kingdom

${ }^{5}$ Department of Psychology,

Virginia Tech, Blacksburg,

Virginia, USA

${ }^{6}$ Fralin Biomedical Research Institute (FBRI) at Virginia Tech Carilion, Roanoke, Virginia, USA

${ }^{7}$ Center for Simulation, Research and Patient Safety, Carilion Clinic, Roanoke, Virginia, USA

Correspondence to

Dr Sandra Keller;

sandra.keller@insel.ch

\begin{abstract}
Objectives To explore predictors and triggers of incivility in medical teams, defined as behaviours that violate norms of respect but whose intent to harm is ambiguous.
\end{abstract}

Design Systematic literature review of quantitative and qualitative empirical studies.

Data sources Database searches according to the Preferred Reporting Items for Systematic Reviews and Meta-Analyses guideline in Medline, CINHAL, PsychInfo, Web of Science and Embase up to January 2020.

Eligibility criteria Original empirical quantitative and qualitative studies focusing on predictors and triggers of incivilities in hospital healthcare teams, excluding psychiatric care.

Data extraction and synthesis of the 1397 publications screened, 53 were included (44 quantitative and 9 qualitative studies); publication date ranged from 2002 to January 2020.

Results Based on the Medical Education Research Study Quality Instrument (MERSQI) scores, the quality of the quantitative studies were relatively low overall (mean MERSQI score of 9.93), but quality of studies increased with publication year $(r=0.52 ; p<0.001)$. Initiators of incivility were consistently described as having a difficult personality, yet few studies investigated their other characteristics and motivations. Results were mostly inconsistent regarding individual characteristics of targets of incivilities (eg, age, gender, ethnicity), but less experienced healthcare professionals were more exposed to incivility. In most studies, participants reported experiencing incivilities mainly within their own professional discipline (eg, nurse to nurse) rather than across disciplines (eg, physician to nurse). Evidence of specific medical specialties particularly affected by incivility was poor, with surgery as one of the most cited uncivil specialties. Finally, situational and cultural predictors of higher incivility levels included high workload, communication or coordination issues, patient safety concerns, lack of support and poor leadership.

Conclusions Although a wide range of predictors and triggers of incivilities are reported in the literature, identifying characteristics of initiators and the targets of incivilities yielded inconsistent results. The use of diverse and high-quality methods is needed to explore the dynamic nature of situational and cultural triggers of incivility.

\section{Strengths and limitations of this study}

To our knowledge, this is the first systematic review on current empirical findings identifying predictors of incivility from both medical and nursing literature.

- To explore the predictors and triggers of incivilities, methods included quantitative and qualitative studies, which allowed an overview of the topic beyond methodological boundaries.

- Examining a wide range of predictors contributes to shed light on which predictors were already extensively investigated and for which predictors more empirical research is needed.

- Overall, the quality of the included studies was low and the conceptualisation of incivility and related terms based mainly on retrospective studies of study participants' perception; this is an inherent limitation to the review.

\section{INTRODUCTION}

- Incivility among healthcare professionals has recently drawn increased attention in the medical world. The potential of incivility to jeopardise optimal patient care-and in turn patient safety, represents one of the major factors that led to their identification as a latent issue in healthcare. ${ }^{12}$ Defined as behaviours that violate norms of respect but whose intent to harm is ambiguous, ${ }^{3}$ incivilities are not typically in the scope of legal sanctions-despite their negative effects. ${ }^{4}$

Healthcare professionals themselves perceive an association between incivilities and decreased patient safety. ${ }^{5}$ For example, a simulation study found a negative effect of rude behaviour on speaking up in medical students. ${ }^{6}$ This result was supported by other simulation studies showing a decrease in communication after the expression of incivilities and also showing negative impact on performance. ${ }^{7}$ In other domains, incivility showed negative effects both on well-being of employees and turnover. ${ }^{8}$

More than three-quarters of healthcare employees have witnessed incivilities by physicians and almost two-thirds incivilities 
by nurses. ${ }^{9}$ In another study, $85 \%$ of the nurses reported having personally experienced incivilities in the past year. ${ }^{10}$ These findings outline the importance and prevalence of the phenomena and the need for additional efforts to reduce frequency and impact. The design of efficient interventions to reduce incivilities is closely tied to an accurate knowledge of the predictors and triggers of incivility in health teams. Predictors are not clearly articulated in the literature and have been explored in a piecemeal fashion. This literature review aims to provide a broad overview of the current empirical knowledge on predictors of incivility.

In this manuscript, we report the results of a systematic review on predictors of incivility in hospitals, including papers up to January 2020. Because a common characteristic of uncivil behaviours is the ambiguity around the intent to harm, ${ }^{311}$ the review investigated closely related and often overlapping terms: incivility, rudeness, disruptive behaviours, interpersonal tensions and the disruptive behaviour part of unprofessional behaviours. These concepts describe impolite and rude conduct ${ }^{12}$ and include overt behaviours such as yelling, ${ }^{13}$ and racial or gender bias. ${ }^{14}$ It also includes more subtle behaviours such as silences, rebukes ${ }^{15}$ gossip and displaced frustration. ${ }^{16}$ Treating others like they are invisible or carelessness by colleagues can also be perceived as incivility. ${ }^{17}$

The medical, and in particular, the nursing literature also uses other terms such as verbal abuse (eg, accusing, blaming, yelling, insulting, humiliating, swearing), ${ }^{13}$ horizontal or lateral violence (ie, violence across members of a same professional group) and bullying, a long-term form of lateral violence ${ }^{18}$ to describe episodes of incivility or violence among health professionals. Because the mechanisms underlying more severe or long-term intrapersonal conflictual behaviours may differ from the ones underlying incivility, we restricted the focus of the present literature review on incivilities and low-intensity aggressive behaviours.

We examined empirical studies that report predictors of incivilities among healthcare teams in hospitals, including physicians, nursing and other professionals involved in patient care in hospitals. We investigated characteristics of both initiators and targets, their professional background and the situational and cultural predictors of incivilities.

\section{METHODS}

The search for literature and the reporting of the results were conducted following the Preferred Reporting Items for Systematic Reviews and Meta-Analyses guidelines. ${ }^{19}$ Quantitative and qualitative studies were included.

\section{Eligibility criteria}

We included original publications of empirical studies focusing on predictors and triggers of incivilities among healthcare hospital teams. Studies conducted with medical or nursing students were included if they

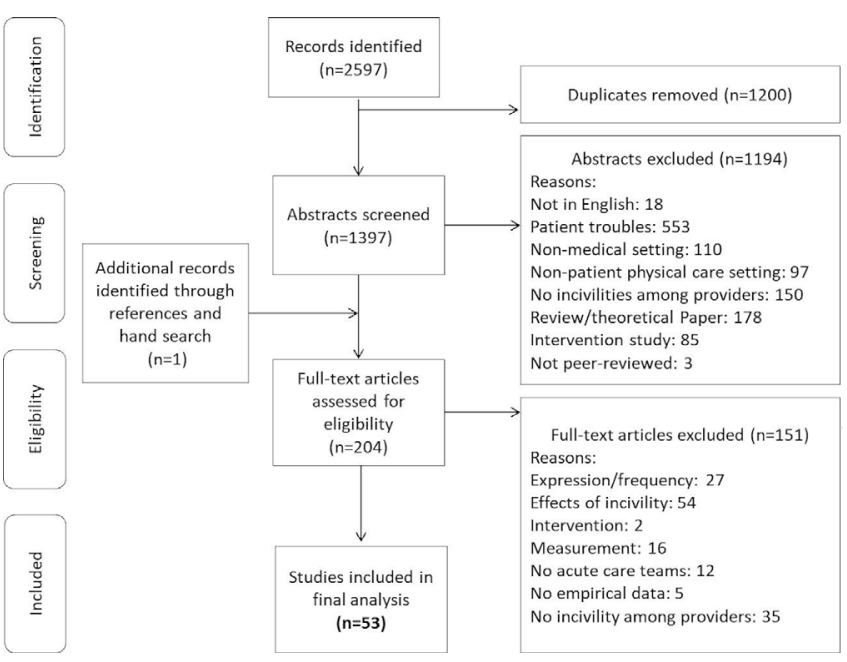

Figure 1 Flow diagram of the selection process of studies included.

focused on clinical experiences of the students. Studies conducted in classroom educational settings were considered as not relevant because we aimed at capturing the dynamics of incivility in the clinical and patient care settings, where time pressure and stress are potentially higher. We included studies related to healthcare professionals working mainly in hospitals, with the exception of psychiatric hospitals. This decision was motivated by the potentially higher prevalence of patient incivility in psychiatric care settings, whereas the focus of this reviews is on incivility within healthcare teams. We set no restrictions in terms of year of publication and searched the full databases up to January 2020, but considered only papers published in English and in peer-reviewed journals with empirical findings related to predictors for incivilities.

\section{Information sources and search strategy}

One author (SK) searched publications in four different databases: Medline, CINHAL, PsychInfo, Web of Science and Embase in January 2020. The search included incivility-related concepts combined with healthcare professions or major services in the hospitals where nonpsychiatric patient care takes place. We followed a systematic search and inclusion-exclusion criteria (figure 1). The Medline database search strategy is included in online supplementary table 1 . We hand searched the references for additional articles.

\section{Study records: data management and selection process}

Publication records were independently extracted from the databases and transferred into an Endnote File. Duplicate articles were excluded. Publication records were then transferred from Endnote to a spreadsheet before coding. A multiple-choice menu was created to code the reasons of exclusion. In a first step, two reviewers (SK and SHP) independently assessed titles and abstracts of the articles for inclusion. All articles potentially reporting empirical original studies on predictors of uncivil behaviours were selected for full-text screening. Divergence in coding were 
resolved by discussion. In a second step, two raters (SK and VZ) screened the full texts to identify studies meeting the inclusion criteria. Again, differences between the two raters were resolved by discussion within the rating team (SK, SHP, VZ). See figure 1 for a schema of the data management process.

\section{Risk of bias}

The quality of quantitative studies was assessed with the Medical Education Research Study Quality Instrument (MERSQI) scale by one author (SK). The MERSQI scale is a validated tool originally designed to assess the quality of medical education publications; it is based on systematic ratings of the study design, sampling, type of data included, validity of measure instruments, data analysis and type of outcome reported..$^{20}$

\section{Synthesis}

*The main goal of the review was to identify the predictors of incivility reported in empirical studies. We categorised the predictors of incivilities reported in the studies into five categories: (i) individual characteristics of initiators of incivilities, (ii) individual characteristics of targets of incivility, (iii) professional groups involved in incivility episodes, in terms of professional background and medical specialisation or hospital department, (iv) situational aspects and (v) cultural determinants. Specific concepts, methods and measurement tools used in the studies were also extracted (table 1).

\section{Patient and public involvement}

It was not appropriate or possible to involve patients or the public in the design, conduct, reporting or dissemination plans of our research.

\section{RESULTS}

The total number of studies selected was 53. We first present descriptive results about the studies, and then discuss their content. Content results are split into initiators, targets, medical specialties, situations and cultural and organisational characteristics.

\section{Descriptive results of the studies \\ Time frame}

Studies meeting the inclusion criteria were published between 2002 and 2020. There was a sharp increase in the number of published studies in 2013, after that the number of published studies remained relatively stable, but on a low frequency level, with four to five published studies per year; since 2018, the number of studies again increased.

\section{Methodology of the included studies}

Forty-four of the 53 studies included quantitative analysis and 9 were based on a qualitative design (table 1).

Among the quantitative studies, the majority, 39 studies, relied on cross-sectional research design and used questionnaires. Other methodologies included analysis of prospective self-reports by the participants (events sampling), ${ }^{21}$ data extracted from or collected in partly with an institutional electronic reporting systems, ${ }^{22-24}$ data collected as part of a physician fitness to practice evaluation programme ${ }^{25}$ or direct observations. ${ }^{26}$

Qualitative studies included four interview studies, ${ }^{27-30}$ one observational study, ${ }^{15}$ one study based on a combination of observations and interviews ${ }^{31}$ and one qualitative analysis of reporting systems. ${ }^{32}$

\section{Quality of studies included}

MERSQI scores, used to assess the quality of the quantitative studies, were relatively low overall, with a mean MERSQI score of 9.93, ranging between 6.5 and 14 on a scale from 5 (lowest possible MERSQI score) to 18 (highest possible MERSQI score) (details of the MERSQI scores for each study are available in online supplementary table 2). More recent publications showed higher MERSQI scores; we found a correlation of $0.52(p<0.001)$ between year of publication and MERSQI scores, see figure 2).

Methodological limitations were often similar across studies. First, many studies relied solely on participants' perceptions, with the exception of four studies based on the evaluation of a fitness to practice evaluation committee,$^{25}$ an expert committee examining the perspectives of multiple professionals involved in a same incivility event, ${ }^{24}$ systematic observations ${ }^{26}$ and an ethnographic observational study. ${ }^{15}$ Second, most questionnaire studies reported low response rates, with a response rate below $50 \%$ in 28 studies. Third, nine studies described prevalence of disruptive behaviours and their triggers, but did not report more complex statistical analyses.

\section{Predictors of incivility}

The results for each subcategory of predictors of incivilities are summarised and the situational and cultural predictors are presented in table 2 .

\section{Initiators of incivility}

When asked about the main triggers of incivilities, healthcare professionals consistently mentioned personality as a major contributor to incivilities or that incivilities were initiated repeatedly by the same individuals. ${ }^{27} 293033-36$ One study showed that personality disorders were indeed more frequently diagnosed in physicians evaluated for disruptive behaviour than physicians evaluated for other issues (eg, sexual harassment). ${ }^{25}$ No other study investigated specific personality characteristics of initiators of incivilities.

Evidence of demographic characteristics of initiators of incivilities was scarce, with one study exploring characteristics of uncivil physicians and two studies exploring the characteristics of uncivil nurses. The only overlapping result across the three studies was that initiators were more likely to be middle-aged or older than their targets. $^{22} 2528$ Two studies found that initiators of incivilities were more likely to belong to the dominant racial 


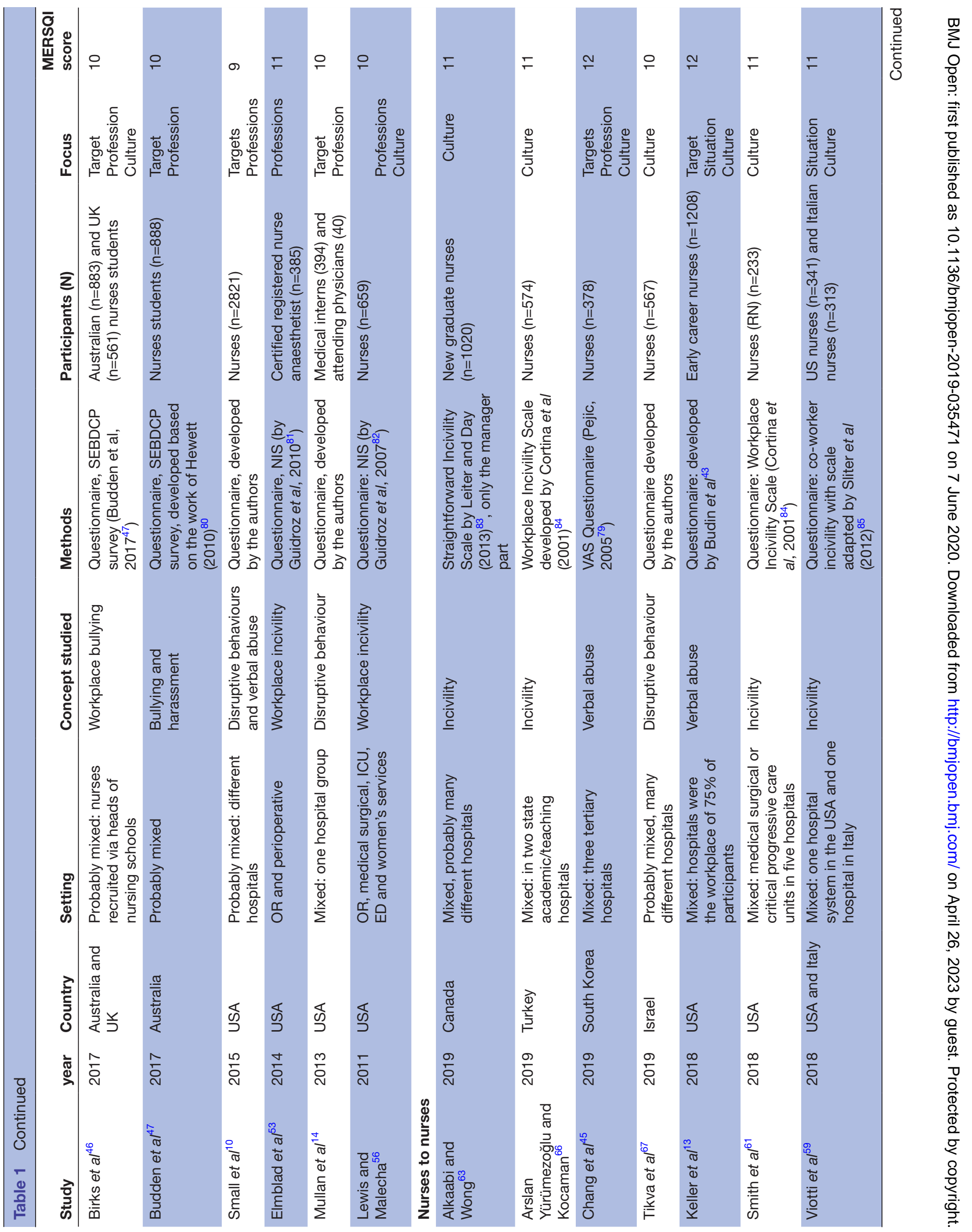




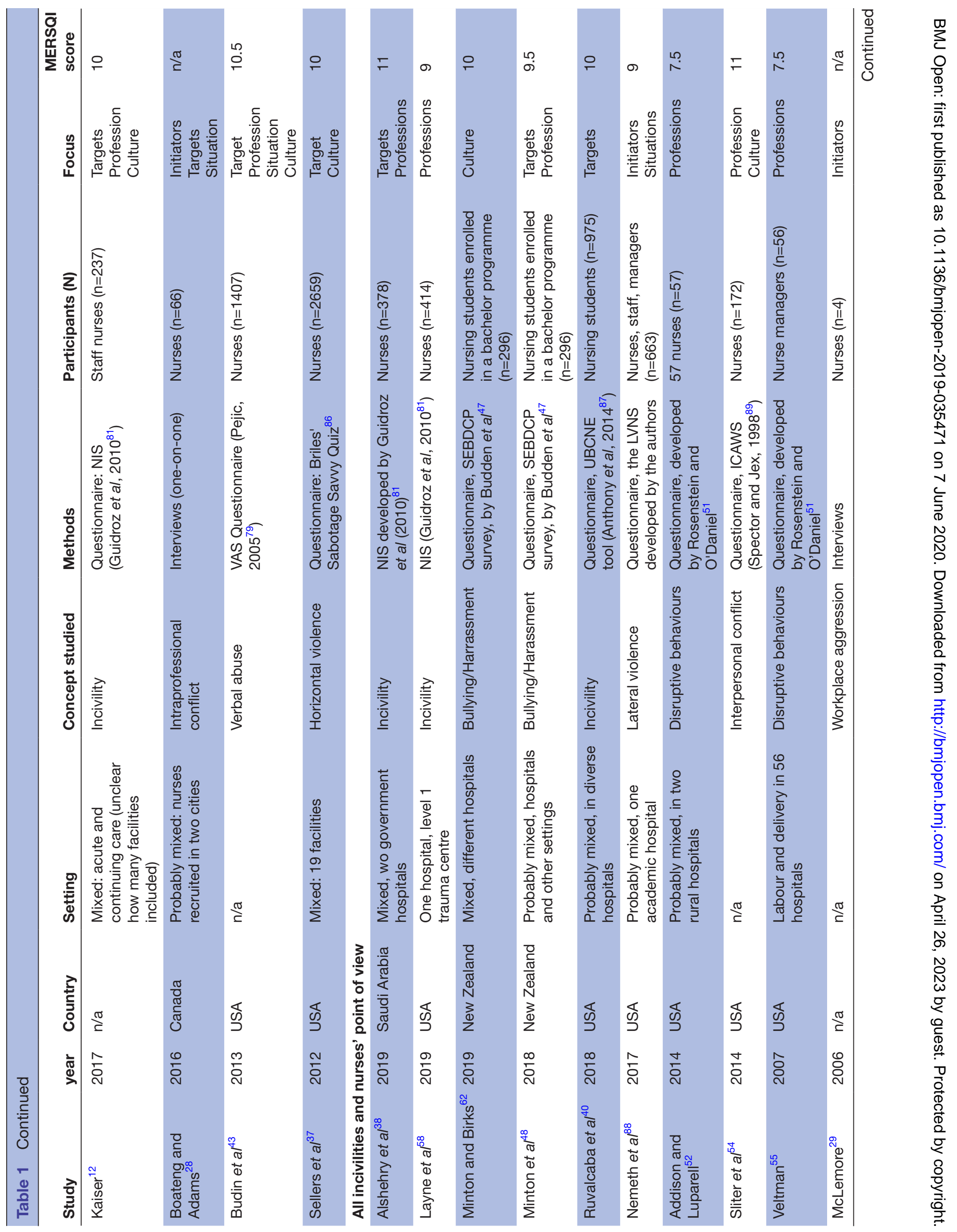




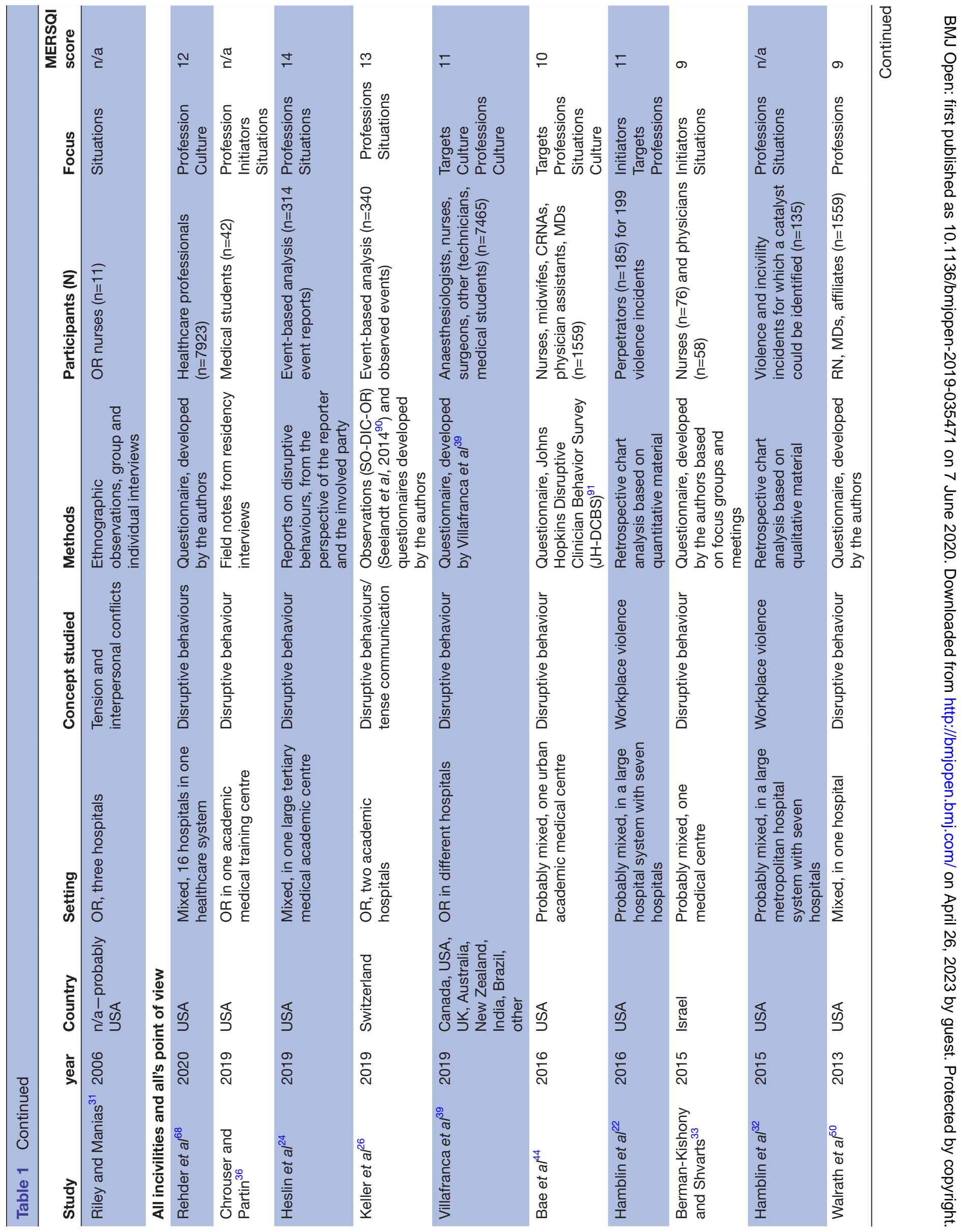



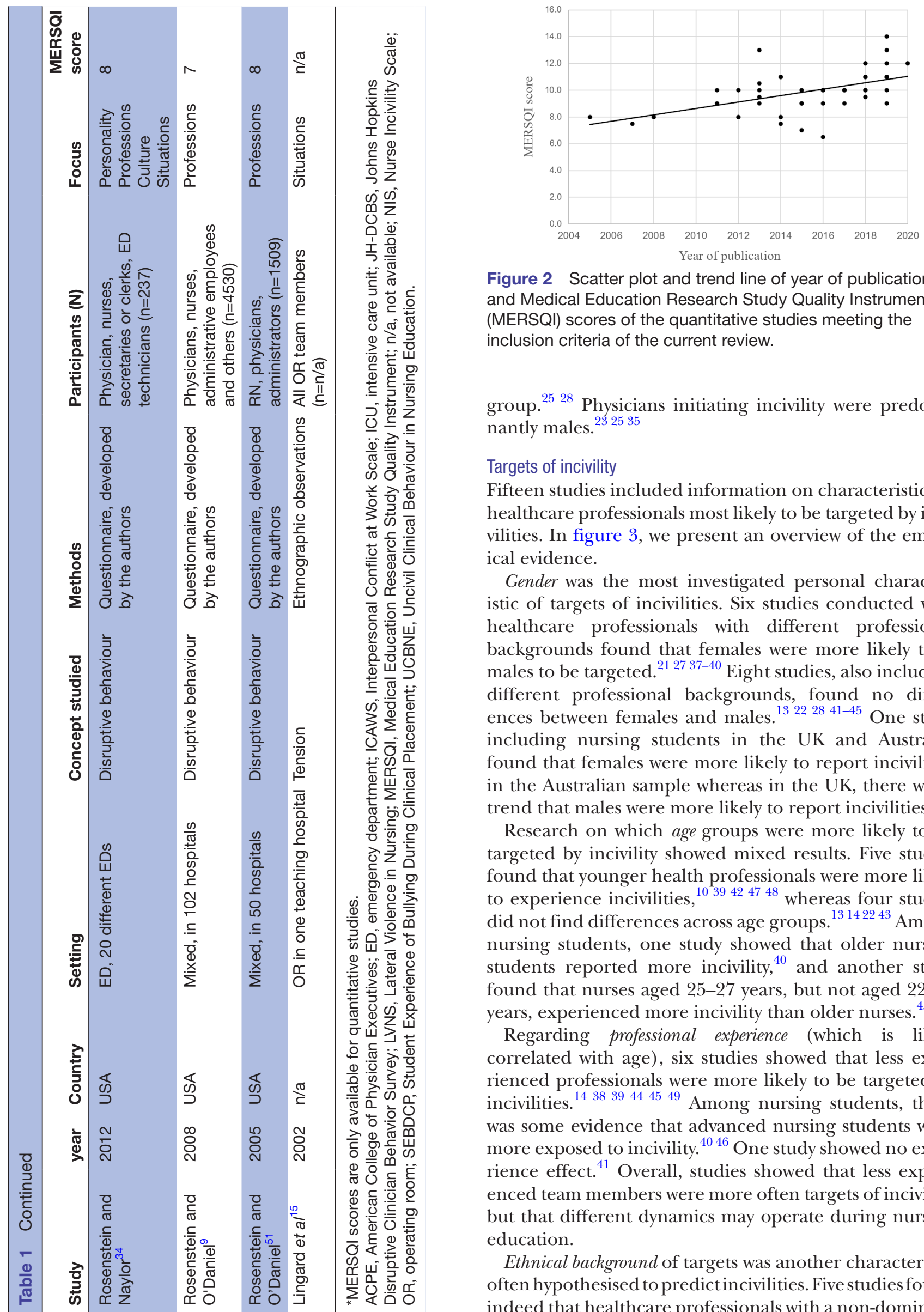

Figure 2 Scatter plot and trend line of year of publication and Medical Education Research Study Quality Instrument (MERSQI) scores of the quantitative studies meeting the inclusion criteria of the current review.

group. ${ }^{25}{ }^{28}$ Physicians initiating incivility were predominantly males. ${ }^{23} 2535$

Targets of incivility

Fifteen studies included information on characteristics of healthcare professionals most likely to be targeted by incivilities. In figure 3, we present an overview of the empirical evidence.

Gender was the most investigated personal characteristic of targets of incivilities. Six studies conducted with healthcare professionals with different professional backgrounds found that females were more likely than males to be targeted. ${ }^{21} 2737-40$ Eight studies, also including different professional backgrounds, found no differences between females and males. ${ }^{132} 28{ }^{41-45}$ One study including nursing students in the UK and Australia, found that females were more likely to report incivilities in the Australian sample whereas in the UK, there was a trend that males were more likely to report incivilities. ${ }^{46}$

Research on which age groups were more likely to be targeted by incivility showed mixed results. Five studies found that younger health professionals were more likely to experience incivilities, ${ }^{10} 39424748$ whereas four studies did not find differences across age groups. ${ }^{1314} 2243$ Among nursing students, one study showed that older nursing students reported more incivility, ${ }^{40}$ and another study found that nurses aged 25-27 years, but not aged 22-24 years, experienced more incivility than older nurses. ${ }^{45}$

Regarding professional experience (which is likely correlated with age), six studies showed that less experienced professionals were more likely to be targeted by incivilities. ${ }^{14} 3839444549$ Among nursing students, there was some evidence that advanced nursing students were more exposed to incivility. ${ }^{40}$ One study showed no experience effect. ${ }^{41}$ Overall, studies showed that less experienced team members were more often targets of incivility, but that different dynamics may operate during nursing education.

Ethnical background of targets was another characteristic often hypothesised to predict incivilities. Five studies found indeed that healthcare professionals with a non-dominant 
Table 2 Situational triggers of incivilities in healthcare teams

\begin{tabular}{|c|c|c|}
\hline Study & Situation & \\
\hline Brewer et $\mathrm{al}^{42}$ & $\begin{array}{l}\text { More physician abuse associated with fewer nurses } \\
\text { working than scheduled. }\end{array}$ & Workload \\
\hline Hamblin et $\left.a\right|^{32}$ & $\begin{array}{l}\text { Work behaviour: unprofessional behaviour, duties } \\
\text { and responsibilities, methods of care, poor } \\
\text { performance. } \\
\text { Work organisation: conflicts about tasks } \\
\text { and procedures, organisational constraints, } \\
\text { interdependence between the workers. }\end{array}$ & $\begin{array}{l}\text { Communication/teamwork } \\
\text { Patient safety } \\
\text { Work responsibilities } \\
\text { Organisational constraints }\end{array}$ \\
\hline Nemeth et $a l^{88}$ & $\begin{array}{l}\text { Most highly causal explanation was stress related } \\
\text { to inadequate staffing or resources, followed by } \\
\text { societal decline in civil behaviour. }\end{array}$ & Workload \\
\hline Viotti et $a l^{59}$ & $\begin{array}{l}\text { Workload as a predictor of incivility only in the USA } \\
\text { but not in the Italian sample. }\end{array}$ & Workload (in one of the study samples) \\
\hline $\begin{array}{l}\text { Berman-Kishony and } \\
\text { Shvarts }^{33}\end{array}$ & $\begin{array}{l}\text { High workload is the second most frequent cause } \\
\text { reported, followed by poor communication, distrust } \\
\text { and disrespect. }\end{array}$ & $\begin{array}{l}\text { Workload } \\
\text { Communication/teamwork }\end{array}$ \\
\hline Budin et $a l^{43}$ & $\begin{array}{l}\text { Higher levels of verbal abuse perceived by nurses } \\
\text { as associated with: fewer nurses working than } \\
\text { scheduled (staffing shortfalls), less perceived } \\
\text { distributive and procedural justice, less promotional } \\
\text { opportunities, more organisational constraints, } \\
\text { higher quantitative workload. }\end{array}$ & Workload \\
\hline Riley and Manias ${ }^{31}$ & $\begin{array}{l}\text { Time: questioning judgement time, controlling } \\
\text { speed, estimating surgeon's time, different } \\
\text { perceptions of time. }\end{array}$ & Time \\
\hline Elhoseny and $\mathrm{Adel}^{60}$ & $\begin{array}{l}\text { Workload as first root cause (reported by } 35 \%), 15 \% \\
\text { reported compensation-related factors. Other: non } \\
\text { work-related situations (12\%). }\end{array}$ & $\begin{array}{l}\text { Workload } \\
\text { Non-work-related factors }\end{array}$ \\
\hline Bradley et $a l^{49}$ & $\begin{array}{l}\text { Doctors describing the situations in which they are } \\
\text { rude: high workload, patient safety compromised, } \\
\text { hierarchy. }\end{array}$ & $\begin{array}{l}\text { Workload } \\
\text { Patient safety }\end{array}$ \\
\hline Lingard et al ${ }^{15}$ & $\begin{array}{l}\text { Time, resources, roles, safety and sterility, situation } \\
\text { control. }\end{array}$ & $\begin{array}{l}\text { Communication/teamwork } \\
\text { Patient safety } \\
\text { Time }\end{array}$ \\
\hline
\end{tabular}


Table 2 Continued

\begin{tabular}{|c|c|c|}
\hline Study & Situation & \\
\hline Bae et $a l^{44}$ & $\begin{array}{l}\text { Triggers of disruptive behaviours at the } \\
\text { interindividual level (eg, questioning providers } \\
\text { about care, lack of teamwork, staff diversity) and } \\
\text { intrapersonal level (eg, lack of competency, fatigue) } \\
\text { related to experienced disruptive behaviours. } \\
\text { Among nurses only (not physicians) organisational } \\
\text { triggers (pressure from high volume, overload, } \\
\text { unresolved issues unit culture) were also predictors } \\
\text { of disruptive behaviours. }\end{array}$ & $\begin{array}{l}\text { Workload } \\
\text { Communication/teamwork } \\
\text { Patient safety } \\
\text { Fatigue }\end{array}$ \\
\hline Shetty et $a l^{21}$ & Consultations with requests for investigations. & Request \\
\hline Heslin et $a l^{24}$ & $\begin{array}{l}\text { Patient factors mentioned as triggers (eg, } \\
\text { challenging anatomy), technical and environmental } \\
\text { factors, organisational factors, stressors (individual } \\
\text { or team). }\end{array}$ & $\begin{array}{l}\text { Workload } \\
\text { Communication/teamwork }\end{array}$ \\
\hline Keller et $a l^{26}$ & $\begin{array}{l}\text { Collaboration and task-related issues were clearly } \\
\text { more frequent sources of tensions than relationship } \\
\text { issues or disagreement about the task. }\end{array}$ & $\begin{array}{l}\text { Communication/teamwork } \\
\text { Task difficulty/stress }\end{array}$ \\
\hline Rehder et $a l^{68}$ & $\begin{array}{l}\text { Disruptive behaviours correlated with poorer } \\
\text { experienced teamwork, lower job satisfaction and } \\
\text { lower perception of management. }\end{array}$ & Communication/teamwork \\
\hline
\end{tabular}

ethnical background or non-native speakers in the country where the study was conducted were more likely to experience incivilities, ${ }^{2728444648}$ whereas four studies did not find differences across ethnic groups. ${ }^{13} 394347$ Of note, two studies found contrasting results with non-native speakers reporting less incivility, ${ }^{40}$ yet in one these studies, nonnative speakers were also unsure about identifying the concept of incivility. ${ }^{48}$

Few studies focused on nurses' educational background $^{1013384144}$ (eg, diploma vs baccalaureate), ${ }^{38}$ shift

Gender
hypothesis: females $>$ males ${ }^{1}$
Age
hypothesis: younger $>$ older $^{2}$
Experience
hypothesis
Ethnicity / language
hypothesis: non majority groups $>$ majority ${ }^{4}$
between groups $\begin{aligned} & \text { Difference in the } \\ & \text { opposite direction }\end{aligned}$
Note. The size of the bubble represents the number of studies included that support the hypothesis,
showed no differences between group respectively showed differences in the opposite direction
${ }^{1}$ Female healthcare professionals may experience more incivility
${ }^{2}$ Younger healthcare professionals may experience more incivility
${ }^{3}$ Healthcare professionals who have less work experienced may experience more incivility
${ }^{4}$ Healhcare professionals who belong to a visible ethnic minority group or are no native speaker may
experience more incivility

Figure 3 Strength of current empirical evidence on the association between characteristics of healthcare professionals and exposure to incivility. type $^{1342}$ or job tenure. ${ }^{2244}$ Cross-sectional studies investigating the association between psychological states such as work satisfaction and incivility are scarce and do not allow to identify consistent results. ${ }^{1343}$

\section{Professional background and medical subspecialties}

Results of the studies included allowed exploration of potential differences in the prevalence of incivilities across medical professions and medical domains. We first report differences across professional backgrounds, for example, nurse and physicians and second, we report comparisons across medical domains (eg, operating room (OR) vs intensive care unit (ICU)).

\section{Professional backgrounds}

The most often examined research question pertained to the prevalence of incivilities in physicians and nurses, and studies investigated the most likely instigator of incivilities among professional groups.

\section{Perception of physicians}

In one study, physicians perceived other physicians as the most frequent initiators of incivilities ${ }^{14}$ and in another study, physicians perceived incivility by other physicians as incivilities having the most negative impact. ${ }^{50}$ Medical interns reported nurses rather than physicians as most frequent initiators of incivilities. ${ }^{14}$ In one study, results were less clear, with physicians perceiving about half of the incivilities initiated by nurses and the other half initiated by physicians. ${ }^{51}$ Nevertheless, slightly more studies 
reported that physicians are the primary source of incivilities to other physicians after training completion.

\section{Perception of nurses}

A majority of studies (seven) found that nurses perceived other nurses as the most frequent or most negative source of incivility, ${ }^{10505253}$ three studies were conducted with nursing students. ${ }^{46-48}$ Four studies reported contrasting results, with physicians perceived as the most frequent source of incivilities by nurses ${ }^{385154}$ or nursing managers. ${ }^{55}$

\section{Studies including professionals from a variety of backgrounds}

Not surprisingly, studies that surveyed diverse medical professionals found mixed results. One study found that physicians were most frequently initiators of incivility, ${ }^{9}$ whereas another study reported similar rates of incivilities by nurses and physicians. ${ }^{34}$ Two studies based on institutional reports found that nurses were more often involved in incivility episodes compared with other professions. ${ }^{22}$ Of note, one of these studies did not include most incivility episodes reported by physicians. ${ }^{22}$ Three OR studies showed contrasting results, with attending surgeons more likely than the other OR healthcare professionals to initiate uncivil episodes. ${ }^{242636}$

Five studies focused on the professional groups most likely to be targeted by incivilities. These studies found that nurses or scrub technicians, ${ }^{26} 39451$ and in general, professions associated with less power in the medical hierarchical system ${ }^{27}$ - more junior surgeons in one study ${ }^{26}$ were more frequently targeted by incivilities.

\section{Medical specialties}

We addressed the question regarding the prevalence of incivilities across specific medical specialties. Surgery or surgical subspecialties appeared in five studies as one of the domains with the most incivilities, compared, for example, with paediatric or emergency departments (EDs),${ }^{12}$ family or internal medicine doctors, ${ }^{25}$ the ICU or medical-surgical units ${ }^{56}$ and other specialties outside radiology and cardiology, ${ }^{49}$ with professionals spending more time in the OR reporting higher incivility levels. ${ }^{39}$ One survey with ICU physicians found contrasting results, showing that surgical specialists were less likely to be uncivil to ICU physicians as compared with non-surgical specialists. ${ }^{57}$ In the same vein, a study found that interactions with surgeons were rated by ED physicians similarly as interactions with other specialists. ${ }^{21}$ Interestingly, in these two latter studies, surgeons were likely to work in other settings than the OR when they interacted with their medical colleagues.

In two studies, radiology appeared to be the specialty associated with the most incivilities. In one study, radiology was followed by general surgery, neurosurgery, cardiology and other specialties ${ }^{49}$ and in the other study radiology was compared with medical, surgical and other specialties. ${ }^{21}$ One study found contrasting results, with radiology as one of the medical domains with the least incivility, for example, compared with surgery, cardiology, trauma and other potentially higher risk specialties. ${ }^{23}$ Other medical domains that were associated with more incivilities were obstetrics ${ }^{12}{ }^{23}$-with one study showing contrasting results, ${ }^{38}$ long term-care, ${ }^{12}$ the ED, ICU, cardiology, ${ }^{23}{ }^{52}$ whereas a study found that nurses working in the ICU reported the least incivilities compared with other nurses. ${ }^{43}$ However, two studies did not find different perceived incivility levels when comparing general, intermediate and ICU, specialty care and nursing clinical support, ${ }^{58}$ respectively general ward, ICU, emergency room and OR. ${ }^{45}$

Three studies that included physicians found that incivilities were more likely during collaboration with other departments compared with participants' own department, ${ }^{23} 4957$ suggesting that intergroup dynamics may also impact incivility. In one of these studies, contradictory results were found for nurses who reported more uncivil behaviours initiated by physicians within their own department than initiated by physicians external to their own departments. ${ }^{23}$

\section{Situational influences on incivilities}

There is evidence that medical professionals report specific situations as fertile grounds for incivilities. We identified seven different situational triggers investigated in different studies and present these results in table 2.

High workload was the most often mentioned trigger of incivilities, reported in ten studies. One questionnaire study did not find an effect of workload, and another study found an effect of workload only in a sample of US nurses but not in a sample of Italian nurses. ${ }^{59}$ The second most frequent situational factors identified as trigger of incivilities are related to the non-technical skills of coordination, communication and teamwork (eg, poor communication, lack of teamwork), reported in nine different studies. Patient safety concerns or poor performance were other factors triggering incivilities reported in three different studies based on ethnographic observations, ${ }^{15}$ retrospective chart analysis ${ }^{32}$ and questionnaires and focus groups. ${ }^{49}$ Two studies found that situations in which healthcare professionals who experienced heavy responsibilities may be more prone for incivilities. In two studies conducted in the OR, time management and negotiations were triggers of tense situations. ${ }^{1531}$

Team composition was also investigated as a potential trigger of incivility, with little familiarity among team members perceived as enhancing incivilities. ${ }^{27} 30$ Finally, organisational constraints, defined as factors preventing employees to perform their task efficiently (eg, because a lack of resources), were perceived as a potential catalyst of incivilities, ${ }^{13} 3236$ as were task difficulties and stress. $^{2636}$

Some other situational factors investigated by a single study and contributing to incivilities in healthcare teams were fatigue,${ }^{44}$ personality conflicts, ${ }^{24}$ the reason for the interaction, that is, request for medical investigations, ${ }^{21}$ compensation or non-work-related factors. ${ }^{60}$ 


\section{Culture and organisation's characteristics}

The relationship of culture, organisation of the department, the hospital or of countries to uncivil behaviour were investigated by different studies. We included results of studies that did not directly measure culture but closely related concepts, such as the impact of department leaders and studies comparing samples of participants working in different countries.

Leadership was associated with incivilities in several studies. Four studies investigating nurses found that the nurses managers' skills to handle incivilities ${ }^{43495661}$ or setting the right tone ${ }^{62}$ was a protective factor against incivilities. A study with physician faculty members found similar results, with participants pointing to the lack of reaction of leaders in handling less severe incivilities. ${ }^{30}$ Furthermore, transformational ${ }^{12}$ or authentic ${ }^{63}$ leadership were found to be protective of incivilities whereas lack of leadership was associated with increased perceived incivility ${ }^{44}$; none of the studies provided data on how transformational leaders contribute to reduced incivility levels. Only one cross-sectional study did not find an association between perceived supervisor support and incivility. ${ }^{13}$

Workplace culture also seems to influence incivilities. For example, three studies found that nurses working in a magnet hospital, a label recognising the quality of nursing care and the professional development of the nursing workforce, ${ }^{64}$ were less likely to experience incivilities. Only one study failed to find an effect ${ }^{13} 65$ and one study found an association between incivility and private founded hospitals. ${ }^{39}$ In three further studies that were conducted with physicians, ${ }^{27} 60$ respectively with a mixed sample of physicians and nurses, ${ }^{34}$ the authors found evidence that culture and training contribute to incivilities, suggesting that uncivil behaviours are learnt and fostered during physicians' training. Furthermore, a positive work culture and support from colleagues or the organisation $^{13436166-68}$ and a diversity climate ${ }^{54}$ were associated with decreased incivilities in seven studies, without evidence of divergent results. In one study, distributive justice, but not procedural justice, was also associated with decreased incivility levels. ${ }^{13}$

Few studies focused on the impact of the countries' cultures on incivilities. Two studies, conducted with nurses, included samples from different countries. One found that the prevalence of incivilities was higher in the USA compared with the Italian nurse sample. The other study compared Australian with UK nurse students and found that Australian nurse students reported more incivility.

\section{DISCUSSION}

This systematic review reports the current state of research related to triggers of uncivil behaviour, reporting consistent and inconsistent findings. Although the interest for this topic has been present for several years in the medical field, the number of studies reporting empirical work only recently started to increase. In addition, the quality scores for most studies, as assessed by MERSQI criteria, were comparable to other samples, ${ }^{20}$ with only three quantitative studies and one qualitative study relying on other measurement methods than perceptions of the study participants. An important result of this review is the need for more empirical research of high quality.

Nevertheless, the existing studies cover a wide range of factors that underlie expression of incivility at work. These predictors or triggers of tensions range from the intrinsic characteristics of the people involved in incivility episodes to situational or cultural aspects influencing the emergence of incivilities. Existing models of incivilities in healthcare teams already include many of the triggers identified empirically, for example, the model of triggers of incivilities in the OR presented by Villafranca et $a t^{69}$ that describes intrapersonal, organisational and interpersonal factors. However, they are not studied in a systematic way.

Studies investigating initiators of incivilities support the influence of personality on uncivil behaviour, sometimes described as 'bad apples'. ${ }^{27}$ However, most of these studies are based on perceptions of study participants. Relatively few studies focused on initiators' perceptions and explored their motivations and interactional context, beyond personality.

Overall, the review shows that demographics of targets are not consistently related to incivilities. Although explored by 15 studies, it was not possible to identify consistent gender differences and specific age and ethnic groups as particularly likely targets of incivilities. However, the studies available on the association between work experience and incivilities show that more experience, often associated with a higher hierarchical status in the organisation, is associated with decreased experience of incivilities. This indicates that higher task proficiency, and higher status, may be protective factors. This finding is in line with the experience of physicians who observed that they were treated with more respect after their promotion to consultant compared with earlier stages of their medical career. $^{49}$

In terms of professional background of tension initiators, the dynamics appeared to be more complex than could be expected. Results showed more evidence of incivilities within similar professional groups, as compared with interprofessional incivilities. Whereas this result is not surprising for physicians, it shows that nurses, rather than physicians, were, in most studies, reported as more likely to initiate incivilities. Of note, most studies did not measure nor control for the frequency of interactions within, and between, professional groups; this is an important potential bias. In addition, most studies are based on the perception of a specific professional group which may also be a source of bias. ${ }^{70}$ The studies also failed to identify consistent differences among medical specialties, with the exception of surgeons during their work in the OR. This result may be explained by the more stressful work conditions, the closer cooperation and the higher risk tasks performed. ${ }^{23}$ 
Different situational aspects influence incivilities in healthcare teams, with workload, communication and teamwork as most important factors, followed by patient safety issues as compared with other predictors. Among cultural factors, leadership and support among the group as well as working in a hospital recognised for excellence in nursing care were among factors recognised as protecting against high incivility levels. Thus, these results suggest that rather than universal professional cultures, local dynamics in specific work situations, departments and hospitals may influence incivilities and should be considered.

Overall, the methodological quality was relatively low for many of the studies reviewed. Methods such as prospective and systematic observation of uncivil interactions ${ }^{152126}$ or relying on hospital surveillance systems ${ }^{22432}$ are rare. Even situational triggers of tensions which need to be studied specifically were investigated with crosssectional survey studies. However, given the only relatively recent interest in this topic, it is important to note that some of the studies included in the review belong to the very first studies that focused on incivilities in healthcare teams. Thus, methodological weaknesses may be offset by the pioneering character of the work, and more recently published papers showed better methodological quality.

\section{STUDY STRENGTHS AND LIMITATIONS \\ Strengths}

One strength of the study was that we included papers based on different methodological approaches to answer the question of the systematic review. This approach allowed to assess similar research questions of studies relying on different methodologies. In addition, this more inclusive approach allows a more extensive overview of the topic.

Because teamwork in healthcare teams is inherently multidisciplinary, we included research conducted with nurses or a mixed population that was often done in nursing science as well as research conducted with physicians, often initiated by physicians. Furthermore, the search process revealed the impressive number of theoretical or position papers (183) on incivilities much more than empirical studies. The high number of theoretical papers is an indicator for the interest in the topic. To understand the phenomenon and what leads to incivilities, there is an urgent need for more empirical research, and in particular research that goes beyond questionnaire studies. Only empirical research can inform the conceptualisation and the understanding of processes triggering incivilities within healthcare teams.

\section{Limitations}

A limitation inherent in the topic of incivility is the conceptualisations of incivilities and related behaviours are subjective, because the intent to harm is per definition ambiguous. ${ }^{3}$ It is thus important to underline that studies that investigate incivility based on perceptions (ie, questionnaire studies) cannot claim to measure incivilities and their triggers beyond participants' perceptions. However, recent studies are promising, showing that perceived incivility can be efficiently assessed with validated tools (see Harris et al for a review) ${ }^{71}$ and methods relying on systematic analysis of institutional reports ${ }^{24}$ or observations $^{26}$ are emerging.

The few studies focusing on the analysis of specific uncivil events rather than perceptions of those events indicate that uncivil behaviour is a complex phenomenon, and much more complex that one initiator behaving in an uncivil way towards a target. ${ }^{1523}$ We did not include conflicts in our search strategy, although conflict behaviour can be uncivil. Conflicts are traditionally defined as caused by divergent opinion on the task or process or caused relationship issues and are of longer term. ${ }^{72}$ Yet, conflicts situations may well underlie uncivil episodes, and further analyses of conflicts in healthcare teams may also contribute to the understanding of uncivil episodes in this context. ${ }^{73} 74$ Similarly, studies that included terms such as horizontal violence, lateral violence, bullying or other forms of aggression without reference to one of our search terms were not included. This allowed to focus the review specifically on less severe forms of rudeness. Yet, there is currently a lack of consistency on the definition of terms related to rude behaviours in the literature. ${ }^{1875}$ We thus cannot exclude that our search strategy did not allow to capture studies that relied on terms usually describing intentional intent to harm (eg, aggression $)^{75}$ and whose definitions widely overlapped with incivility in individual works.

\section{CONCLUSION}

Given the known impact of incivilities on both patient care processes ${ }^{7}$ and healthcare professionals' health, ${ }^{7677}$ the need for efficient interventions to reduce incivilities in healthcare teams is likely to increase. Such interventions need to be based on empirical evidence. The present systematic review showed that most studies investigated general characteristics of initiators and targets of incivilities. Situational aspects that foster incivilities are clearly understudied, so we may underestimate the probability that incivilities are a result of coordination problems. Further studies should concentrate on these situational triggers (cooperation, task requirements). Future incivility research in the medical field also needs to adopt higher quality methods than current studies. Only if these two conditions are satisfied can empirical results then inform the design of interventions to reduce incivility and the potential harm to providers and patients. Interventions at the organisational level are particularly likely to benefit from this research since healthcare organisations can influence to a certain degree the design of work processes, leadership within departments and cultural aspects that tackle rather than promote incivility. 
Acknowledgements The authors would like to thank Rita McCandless and Erin M. Smith, PhD, for their technical support and advice about the library resources and giving us access to the full texts that could not be retrieved by the authors. The authors would like to thank Franziska Tschan, PhD for her critical review of an earlier version of the manuscript.

Contributors Study design: SK, SHP and SY. Data analysis: SK, VZ and SHP. Drafting the work or critically revising it: SK, SY, VZ and SHP.

Funding This work was sponsored by a grant of the Swiss National Science Foundation (grant number P2NEP1 178574).

Competing interests None declared.

Patient and public involvement Patients and/or the public were not involved in the design, conduct, reporting or dissemination plans of this research.

Patient consent for publication Not required.

Provenance and peer review Not commissioned; externally peer reviewed.

Data availability statement All data relevant to the study are included in the article or uploaded as supplementary information.

Open access This is an open access article distributed in accordance with the Creative Commons Attribution 4.0 Unported (CC BY 4.0) license, which permits others to copy, redistribute, remix, transform and build upon this work for any purpose, provided the original work is properly cited, a link to the licence is given, and indication of whether changes were made. See: https://creativecommons.org/ licenses/by/4.0/

Author note Sandra Keller currently works at Bern University Hospital (Inselspital), Switzerland.

ORCID iD

Sandra Keller http://orcid.org/0000-0003-3229-9003

\section{REFERENCES}

1 Leape LL, Shore MF, Dienstag JL, et al. Perspective: a culture of respect, part 1: the nature and causes of disrespectful behavior by physicians. Acad Med 2012;87:845-52.

2 Flin R. Rudeness at work. BMJ 2010;340:c2480.

3 Andersson LM, Pearson CM. Tit for Tat? The spiraling effect of incivility in the workplace. Acad Manage Rev 1999;24:452-71.

4 Shapiro J. Confronting unprofessional behaviour in medicine. BMJ 2018;360:k1025.

5 Rosenstein AH, O'Daniel M. Impact and implications of disruptive behavior in the perioperative arena. J Am Coll Surg 2006;203:96-105.

6 Barzallo Salazar MJ, Minkoff H, Bayya J, et al. Influence of surgeon behavior on trainee willingness to speak up: a randomized controlled trial. J Am Coll Surg 2014;219:1001-7.

7 Riskin A, Erez A, Foulk TA, et al. The impact of rudeness on medical team performance: a randomized trial. Pediatrics 2015;136:487-95.

8 Porath CL, Pearson CM. Emotional and behavioral responses to workplace incivility and the impact of hierarchical status. J App/ Soc Psychol 2012;42:E326-57.

9 Rosenstein $\mathrm{AH}, \mathrm{O}^{\prime}$ Daniel M. A survey of the impact of disruptive behaviors and communication defects on patient safety. Jt Comm J Qual Patient Saf 2008;34:464-71.

10 Small CR, Porterfield S, Gordon G. Disruptive behavior within the workplace. Appl Nurs Res 2015;28:67-71.

11 Hershcovis MS, Barling J. Towards a multi-foci approach to workplace aggression: a meta-analytic review of outcomes from different perpetrators. J Organ Behav 2010;31:24-44.

12 Kaiser JA. The relationship between leadership style and nurse-tonurse incivility: turning the lens inward. J Nurs Manag 2017;25:110-8.

13 Keller R, Krainovich-Miller B, Budin W, et al. Predictors of nurses' experience of verbal abuse by nurse colleagues. Nurs Outlook 2018;66:190-203.

14 Mullan CP, Shapiro J, McMahon GT. Interns' experiences of disruptive behavior in an academic medical center. J Grad Med Educ 2013;5:25-30.

15 Lingard L, Reznick R, Espin S, et al. Team communications in the operating room: talk patterns, sites of tension, and implications for novices. Acad Med 2002;77:232-7.

16 Oja KJ. Incivility and professional Comportment in critical care nurses. AACN Adv Crit Care 2017;28:345-50.

17 Abdollahzadeh F, Asghari E, Doshmangir L, et al. Workplace Incivility as an extensively used, but seldom defined concept in nursing. Nurs Midwifery Stud 2017;6:e41029.
18 Bambi S, Foà C, De Felippis C, et al. Workplace incivility, lateral violence and bullying among nurses. A review about their prevalence and related factors. Acta Biomed 2018;89:51.

19 Moher D, Shamseer L, Clarke M, et al. Preferred reporting items for systematic review and meta-analysis protocols (PRISMA-P) 2015 statement. Syst Rev 2015;4:1.

20 Reed DA, Cook DA, Beckman TJ, et al. Association between funding and quality of published medical education research. JAMA 2007;298:1002-9.

21 Shetty AL, Vaghasiya M, Boddy R, et al. Perceived incivility during emergency department phone consultations. Emerg Med Australas 2016;28:256-61.

22 Hamblin LE, Essenmacher L, Ager J, et al. Worker-to-Worker violence in hospitals: perpetrator characteristics and common dyads. Workplace Health Saf 2016;64:51-6.

23 Goettler CE, Butler TS, Shackleford P, et al. Physician behavior: not ready for 'Never'land. Am Surg 2011;77:1600-5.

24 Heslin MJ, Singletary BA, Benos KC, et al. Is disruptive behavior inherent to the surgeon or the environment? Analysis of 314 events at a single academic medical center. Ann Surg 2019;270:463-72.

25 Finlayson AJR, Dietrich MS, Neufeld R, et al. Restoring professionalism: the physician fitness-for-duty evaluation. Gen Hosp Psychiatry 2013;35:659-63.

26 Keller S, Tschan F, Semmer NK, et al. "Disruptive behavior" in the operating room: A prospective observational study of triggers and effects of tense communication episodes in surgical teams. PLoS One 2019;14:e0226437

27 Cochran A, Elder WB. A model of disruptive surgeon behavior in the perioperative environment. J Am Coll Surg 2014;219:390-8.

28 Boateng GO, Adams TL. "Drop dead ... I need your job": An exploratory study of intra-professional conflict amongst nurses in two Ontario cities. Soc Sci Med 2016;155:35-42.

29 McLemore MR. Workplace aggression: beginning a dialogue. Clin J Oncol Nurs 2006;10:455-6.

30 Pattani R, Ginsburg S, Mascarenhas Johnson A, et al. Organizational factors contributing to incivility at an academic medical center and systems-based solutions: a qualitative study. Acad Med 2018;93:1569-75.

31 Riley R, Manias E. Governing time in operating rooms. J Clin Nurs 2006;15:546-53.

32 Hamblin LE, Essenmacher L, Upfal MJ, et al. Catalysts of worker-to-worker violence and incivility in hospitals. J Clin Nurs 2015;24:2458-67.

33 Berman-Kishony T, Shvarts S. Universal versus tailored solutions for alleviating disruptive behavior in hospitals. Isr J Health Policy Res 2015;4:12.

34 Rosenstein $\mathrm{AH}$, Naylor B. Incidence and impact of physician and nurse disruptive behaviors in the emergency department. $J$ Emerg Med 2012;43:139-48.

35 Bansal AS. Disruptive behaviour amongst doctors, myth or reality? J Evol Med Dent Sci 2014;3:399-406.

36 Chrouser KL, Partin MR. Intraoperative disruptive behavior: the medical student's perspective. J Surg Educ 2019;76:1231-40.

37 Sellers KF, Millenbach L, Ward K, et al. The degree of horizontal violence in rns practicing in New York state. J Nurs Adm 2012;42:483-7.

38 Alshehry AS, Alquwez N, Almazan J, et al. Workplace incivility and its influence on professional quality of life among nurses from multicultural background: a cross-sectional study. J Clin Nurs 2019:28:2553-64.

39 Villafranca A, Hiebert B, Hamlin C, et al. Prevalence and predictors of exposure to disruptive behaviour in the operating room. Can $J$ Anaesth 2019;66:781-94.

40 Ruvalcaba JG, Welch S, Carlisle J. ESL versus Non-ESL nursing students' perceptions of Incivility in the clinical setting. J Nurs Educ 2018;57:720-6.

41 Heydari A, Rad M, Rad M. Evaluating the Incivility between staff nurses and Matrons employed in Iran. Acta Fac Medicae Naiss 2015;32:137-46

42 Brewer CS, Kovner CT, Obeidat RF, et al. Positive work environments of early-career registered nurses and the correlation with physician verbal abuse. Nurs Outlook 2013;61:408-16.

43 Budin WC, Brewer CS, Chao Y-Y, et al. Verbal abuse from nurse colleagues and work environment of early career registered nurses. $J$ Nurs Scholarsh 2013;45:308-16.

44 Bae S-H, Dang D, Karlowicz KA, et al. Triggers contributing to health care clinicians' disruptive behaviors. J Patient Saf 2016. doi:10.1097/ PTS.0000000000000288. [Epub ahead of print: 02 Nov 2016].

45 Chang HE, Park MY, Jang H, et al. Relationships among demands at work, aggression, and verbal abuse among registered nurses in South Korea. Nurs Outlook 2019;67:567-77. 
46 Birks M, Cant RP, Budden LM, et al. Uncovering degrees of workplace bullying: a comparison of baccalaureate nursing students experiences during clinical placement in Australia and the UK. Nurse Educ Pract 2017;25:14-21.

47 Budden LM, Birks M, Cant R, et al. Australian nursing students' experience of bullying and/or harassment during clinical placement. Collegian 2017;24:125-33.

48 Minton C, Birks M, Cant R, et al. New Zealand nursing students' experience of bullying/harassment while on clinical placement: A cross-sectional survey. Collegian 2018;25:583-9.

49 Bradley V, Liddle S, Shaw R, et al. Sticks and stones: investigating rude, dismissive and aggressive communication between doctors. Clin Med 2015;15:541-5.

50 Walrath JM, Dang D, Nyberg D. An organizational assessment of disruptive clinician behavior: findings and implications. J Nurs Care Qual 2013;28:110-21.

51 Rosenstein AH, O'Daniel M. Original Research: Disruptive Behavior and Clinical Outcomes: Perceptions of Nurses and Physicians: Nurses, physicians, and administrators say that clinicians' disruptive behavior has negative effects on clinical outcomes. AJN Am J Nurs 2005;105:54-64.

52 Addison K, Luparell S. Rural Nurses' Perceptions of Disruptive Behavior and Clinical Outcomes: A Pilot Study. OJRNHC 2014:14:66-82.

53 Elmblad R, Kodjebacheva G, Lebeck L. Workplace incivility affecting cRNAs: a study of prevalence, severity, and consequences with proposed interventions. AANA J 2014:82:437-45.

54 Sliter M, Boyd E, Sinclair R, et al. Inching toward Inclusiveness: diversity climate, interpersonal conflict and well-being in women nurses. Sex Roles 2014:71:43-54.

55 Veltman LL. Disruptive behavior in obstetrics: a hidden threat to patient safety. Am J Obstet Gynecol 2007;196:587.e1-587.e5.

56 Lewis PS, Malecha A. The impact of workplace incivility on the work environment, manager skill, and productivity. J Nurs Adm 2011;41:41-7.

57 Klingberg K, Gadelhak K, Jegerlehner SN, et al. Bad manners in the emergency department: Incivility among doctors. PLoS One 2018; $13: 11$

58 Layne DM, Anderson E, Henderson S. Examining the presence and sources of incivility within nursing. J Nurs Manag 2019;27:1505-11.

59 Viotti S, Converso D, Hamblin LE, et al. Organisational efficiency and co-worker incivility: a cross-national study of nurses in the USA and Italy. J Nurs Manag 2018;26:597-604.

60 Elhoseny TA, Adel A. Disruptive physician behaviors and their impact on patient care in a health insurance hospital in Alexandria, Egypt. $J$ Egypt Public Health Assoc 2016;91:80-5.

61 Smith JG, Morin KH, Lake ET. Association of the nurse work environment with nurse incivility in hospitals. J Nurs Manag 2018;26:219-26.

62 Minton C, Birks M. "You can't escape it": Bullying experiences of New Zealand nursing students on clinical placement. Nurse Educ Today 2019;77:12-17.

63 Alkaabi O, Wong C. Relationships among authentic leadership, manager incivility and trust in the manager. Leadersh Health Serv 2019;33:27-42

64 Kramer M, Schmalenberg C. Magnet hospitals: Part I. Institutions of excellence. J Nurs Adm 1988:18:13-24.

65 American Nurses Association. ANCC magnet recognition program $®$. Available: https://www.nursingworld.org/organizational-programs/ magnet/ [Accessed 1 Nov 2019].

66 Arslan Yürümezoğlu H, Kocaman G. Structural empowerment, workplace incivility, nurses' intentions to leave their organisation and profession: a path analysis. J Nurs Manag 2019;27:732-9.

67 Tikva SS, Kluger AN, Lerman Y. Disruptive behaviors among nurses in Israel - association with listening, wellbeing and feeling as a victim: a cross-sectional study. Isr J Health Policy Res 2019;8:1-9.

68 Rehder KJ, Adair KC, Hadley A, et al. Associations between a new disruptive behaviors scale and teamwork, patient safety, work-life balance, burnout, and depression. Jt Comm J Qual Patient Saf 2020;46:18-26
69 Villafranca A, Hamlin C, Enns S, et al. Disruptive behaviour in the perioperative setting: a contemporary review. Can J Anaesth 2017;64:128-40.

70 Müller P, Tschan F, Keller S, et al. Assessing perceptions of teamwork quality among perioperative team members. AORN J 2018;108:251-62.

71 Harris WC, Usseglio J, Chapman-Rodriguez R, et al. A scoping review of validated tools to measure Incivility in healthcare settings. $J$ Nurs Adm 2019;49:447-53.

72 Jehn KA. A qualitative analysis of conflict types and dimensions in organizational groups. Adm Sci Q 1997;42:530-57.

73 Bochatay N, Bajwa NM, Cullati S, et al. A multilevel analysis of professional conflicts in health care teams: insight for future training. Acad Med 2017;92:S84-92.

74 Katz JD. Conflict and its resolution in the operating room. J Clin Anesth 2007;19:152-8.

75 Hills DJ. Defining and classifying aggression and violence in health care work. Collegian 2018;25:607-12.

76 Wing T, Regan S, Spence Laschinger HK. The influence of empowerment and incivility on the mental health of new graduate nurses. J Nurs Manag 2015;23:632-43.

77 Babenko-Mould Y, Laschinger HKS. Effects of incivility in clinical practice settings on nursing student burnout. Int J Nurs Educ Scholarsh 2014;11:145-54.

78 MacDonald O. Disruptive physician behavior, 2011. Quantia MD, American College of physician executives. Available: http://www. quantiamd.com/q-qcp/Disruptive_Physician_Behavior.pdf [Accessed 1 November 2013]

79 Pejic AR. Verbal abuse: a problem for pediatric nurses. Pediatr Nurs 2005;31:271-9.

80 Hewett D. Workplace Violence Targeting Student Nurses in the Clinical Areas. South Africa, Stellenbosch University, 2010.

81 Guidroz AM, Burnfield-Geimer JL, Clark O, et al. The nursing incivility scale: development and validation of an occupation-specific incivility measure. J Nurs Meas 2010;18:176-200.

82 Guidroz AM, Burnfield JL, Clark OL. The nursing incivility scale: development of an occupation-specific incivility measure. Poster presented at the 22nd Annual Conference of the Society for Industrial and Organizational Psychology; May, New York, NY., 2007.

83 Leiter MP, Day A. Straightforward Incivility scale manual, technical document. Wolfville Centre for Organizational Research, Acadia University; 2013

84 Cortina LM, Magley VJ, Williams JH, et al. Incivility in the workplace: incidence and impact. J Occup Health Psychol 2001;6:64-80.

85 Sliter M, SliterK, Jex S. The employee as a punching bag: the effect of multiple sources of incivility on employee withdrawal behavior and sales performance. J Organ Behav 2012;33:121-39.

86 Briles J. The Briles report on women in healthcare: changing conflict to collaboration in a toxic workplace. San Francisco,CA: JosseyBass, 1994

87 Anthony M, Yastik J, MacDonald DA, et al. Development and validation of a tool to measure incivility in clinical nursing education. $J$ Prof Nurs 2014;30:48-55.

88 Nemeth L, Stanley K, Martin M, et al. Lateral violence in nursing survey: instrument development and validation. Health Care 2017;5:12.

89 Spector PE, Jex SM. Development of four self-report measures of job stressors and strain: interpersonal conflict at work scale, organizational constraints scale, quantitative workload inventory, and physical symptoms inventory. J Occup Health Psychol 1998;3:356-67.

90 Seelandt JC, Tschan F, Keller S, et al. Assessing distractors and teamwork during surgery: developing an event-based method for direct observation. BMJ Qual Saf 2014;23:918-29.

91 Dang D, Nyberg D, Walrath JM, et al. Development and validation of the Johns Hopkins disruptive clinician behavior survey. Am J Med Qual 2015;30:470-6. 